\title{
Betrayal Intention in International Business Relationships: Temporal and Contractual Moderating Effects (An Abstract)
}

\author{
Leonidas C. Leonidou, Bilge Aykol, Thomas A. Fotiadis, \\ and Paul Christodoulides
}

\begin{abstract}
Betrayal is a very common, but relatively under-researched, dark side phenomenon in interfirm relationships that warrants investigation. We propose a conceptual model of the factors reducing betrayal intention in exporter-importer (E-I) working relationships and its resulting effect on actual betrayal. Using a random sample of 262 indigenous exporters of manufactured goods based in Greece, we confirm that betrayal intention in their relationships with foreign buyers is significantly and positively affected by four key parameters, namely, the existence of low trust, limited communication, absence of long-term orientation, and loose social bonds. An importer's betrayal intention is subsequently very likely to develop into actual betrayal in the relationship. However, this likelihood is lower in the case of older relationships, as well as those characterized by contractual obligation between the interacting parties.
\end{abstract}

\footnotetext{
L.C. Leonidou

University of Cyprus, Nicosia, Cyprus

e-mail: leonidas@ucy.ac.cy

B. Aykol ( $\square)$

Dokuz Eylül University, İzmir, Turkey

e-mail: bilge.aykol@deu.edu.tr

T.A. Fotiadis

Democritus University of Thrace, Xanthi, Greece

e-mail: thomas.fotiadis@yahoo.gr

P. Christodoulides

Cyprus University of Technology, Limassol, Cyprus

e-mail: paul.christodoulides@cut.ac.cy
} 